\title{
ACCIÓN INTERCULTURAL EN LOS ÁMBITOS DE LA CALIDAD DE VIDA
}

\author{
OCTAVIO VÁZQUEZ, PATRICIA VAZ, MANUELA FERNÁNDEZ, \\ MIRIAN FERNÁNDEZ, JOSÉ CARLOS LEÓN \\ Universidad de Huelva (España).
}

RESUMEN

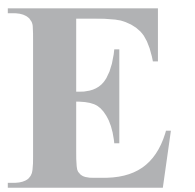

1 fenómeno de la inmigración y de la diversidad cultural está cada vez más presente en las sociedades actuales; el abordaje de esta pluralidad cultural desde un enfoque intercultural se hace necesario para favorecer la convivencia. Este artículo refleja las acciones y las bases teóricas del proyecto "Interculture map", desarrollado a nivel europeo por socios de diversos países como Italia, Bélgica, República Checa y España, con el fin de establecer un mapa de las acciones interculturales en Europa y de realizar una aproximación conceptual a la noción de Buena Práctica intercultural.

Palabras clave: interculturalidad, inmigración, estudios de caso, calidad de vida, Europa.

\section{ABSTRACT: Intercultural Actions Related to Quality of Life}

The phenomena of immigration and cultural diversity are increasingly present in contemporary society. In the interests of promoting co-existence, this cultural plurality is necessarily addressed here from an intercultural approach. This article reflects the actions and theoretical bases behind the "Interculture Map" project, a European project with partners in countries such as Italy, Belgium, the Czech Republic and Spain which aims to create a map of intercultural actions in Europe and to identify a conceptual approach to the idea of Good Intercultural Practice.

Key words: interculture, migrants, case studies, quality of life, Europe.

CORRESPONDENCIA:

Octavio Vázquez Aguado

Departamento de Sociología y Trabajo Social. Universidad de Huelva.

Campus de «El Carmen» Carretera de Palos de la Frontera s/n. Avenida de las

Fuerzas Armadas, s/n. 21071 Huelva. España.

Octavio@uhu.es 


\section{INTRODUCCIÓN}

El presente artículo refleja parte de los resultados obtenidos por el grupo Ágora de la Universidad de Huelva en el marco del proyecto Interculture Map, que analiza las buenas prácticas interculturales en la Europa actual con el propósito de definir las características que deben poseer para servir tanto de guía a futuras actuaciones interculturales como de referencia a posibles marcos legislativos en desarrollo. En el proyecto han participado el Multicultural Center de Praga (MKC), el Centre Bruxellois de Action Interculturelle (CBAI), Africa e Mediterraneo-A\&M- (Bolonia) y el Grupo de Investigación Ágora de la Universidad de Huelva. Ha sido financiado por la iniciativa europea INTI (JLS/2004/INTI153). Su periodo de ejecución ha transcurrido entre junio de 2005 y finales de 2006. Los objetivos del proyecto estaban centrados en definir un mapa de buenas prácticas interculturales en Europa en diferentes ámbitos de actuación: aspectos mediáticos $(M K P)$, artísticos $(A \& M)$, educativos ( $C B A I$ ) y calidad de vida (empleo, alojamiento y salud) -objeto de análisis del grupo Ágora-. Antes de detallar las diferentes acciones desarrolladas, creemos necesario hacer una breve aclaración sobre lo que entendemos por acción intercultural y calidad de vida.

\subsection{Los referentes teóricos: acción intercultural y calidad de vida}

Siguiendo a Vázquez (2005: 115-116), definimos la acción intercultural como un proceso que comienza con la constatación de la diversidad que existe sobre un territorio. Afecta a toda la población y no se dirige, por tanto, exclusivamente a los miembros de los grupos minoritarios o a los de origen extranjero. Tiene una dimensión preventiva en la medida en la que puede facilitar a los individuos concernidos elementos que les ayuden a manejar su convivencia en la diversidad. Posee, asimismo, una función reparadora de la estructura social, puesto que sus estrategias se desarrollan también en situaciones de conflicto. Nos impulsa al reconocimiento del otro en su singularidad (Burdalo, 2006), sin negar por ello la existencia de conflictos derivados de la coexistencia sobre un mismo territorio de individuos socioculturalmente diferenciados.

Pero no debemos olvidar que, en muchas sociedades, actualmente la diferencia es entendida como causa de divergencia, y asumida como amenaza del propio sistema social, ideológico, económico, político, religioso o incluso identitario. En los países receptores de inmigración, a menudo, se considera que los inmigrantes suponen una amenaza para "nuestros" empleos, rentas, cultura, orden público y estilos de vida (Triandafyllidou, 2005: 45). Desde esta óptica, la relación que se establece con el extraño/forastero genera en palabras de Gudykunst (en Vázquez, 2005: 112-113) incertidumbre y ansiedad, siendo lo diverso entendido como algo negativo, lo que genera prejuicios y actitudes discriminatorias hacia las minorías.

¿Cómo repercute el concepto de calidad de vida en la acción intercultural? La calidad de vida es una constructo social de carácter multidimensional y abordaje interdisciplinar que alude a condiciones tanto objetivas como subjetivas de integración social y desarrollo del individuo (Schalock, Brown y cols., 2002:7-8). Lo subjetivo es la evaluación que el individuo realiza de las condiciones objetivas de su situación, mientras que lo objetivo, queda defini- 
do, según Blanco (1998: 65), por los recursos de que dispone el individuo para controlar y dirigir su propia vida. La dimensión subjetiva de la calidad de vida es posible cuando los individuos tienen atendidas la dimensión objetiva.

Cada una de las áreas objeto de estudio de la calidad de vida (empleo, salud y alojamiento) refieren derechos humanos fundamentales y han de darse por fuerza cuando individuos de diverso trasfondo cultural interactúan, puesto que la salud es una propiedad inherente al ser humano, y el trabajo y el alojamiento son factores sociales que garantizan la existencia. Estas áreas constituyen la base de la infraestructura social sin la que no puede desarrollarse el resto de garantías sociales, son condiciones básicas y de intervención de primer orden para la supervivencia y desarrollo de una vida digna de los individuos.

Realizar actuaciones sociales en materia de empleo desde un enfoque intercultural facilitará una mejor adaptación del individuo al puesto de trabajo, mejorando su productividad y contribución a la sociedad de acogida. Esto incide, a su vez, en la percepción positiva que la comunidad de acogida pueda tener de las diferencias culturales que identifican a los individuos, incrementando sus posibilidades de integración en dicha comunidad.

La falta de trabajo dificulta el acceso a una vivienda, al no contar con ingresos derivados del desarrollo de una actividad laboral. Carecer de alojamiento priva al individuo de su espacio privado, exponiéndolo a un espacio público que en muchas ocasiones impide su aceptación. A esto hay que añadir las diferentes pautas culturales en relación a la vivienda que hacen aún más complicado el acceso a la misma y la convivencia.

Por otra parte, no tener acceso a las condiciones básicas de higiene, descanso y protección hace al colectivo inmigrante vulnerable socialmente. La noción de salud no es la misma en todas las culturas, puesto que lo que unos consideran inadecuado es aceptable o incluso deseable para otros. Esto puede aplicarse a los hábitos alimenticios, al olor corporal, etc.; aspectos cotidianos que se ven muy influenciados por las pautas culturales.

La consideración de las peculiaridades de identidad cultural en el ámbito sanitario y la aplicación de estrategias de comunicación intercultural facilitan una mejor atención al paciente, ayudan a entender mejor su situación y padecimiento e incluso a ajustar mejor el tratamiento.

Los sujetos sin trabajo, vivienda o con malas condiciones de salud son percibidos por la comunidad de acogida como desestabilizadores del equilibrio social y posibles potenciadores de su ruptura. Las intervenciones sociales desde lo intercultural en estas áreas deben ir encaminadas a la consecución de la integración social de aquéllos que son excluidos por su diferencia y a conducir hacia un espacio de convivencia en el que, a través del diálogo intercultural, sea posible trascender las situaciones asimétricas, eliminando elementos de esta exclusión por discriminación de la diferencia (Arpini, 2005: 70).

\subsection{La metodología del proyecto: estudios de caso}

El trabajo de campo del proyecto Interculture Map comenzó con la creación de una base de datos de acciones interculturales en Europa construida por el conjunto de los socios participantes en este proyecto. En nuestro caso, la 
participación tuvo una triple dimensión: en primer lugar, la elaboración de un listado con 200 iniciativas españolas en el ámbito de la interculturalidad. En segundo lugar, de este listado inicial, la selección de un total de 20 proyectos relativos a otros ámbitos de investigación del proyecto como educación, formación, medios de comunicación, arte, etc. Finalmente, nos centramos en la localización de 50 proyectos de buenas prácticas relacionados con el ámbito de la calidad de vida (empleo, alojamiento y salud) con información relativa al país, área, organización promotora, web, mail, dirección, teléfono, fax y año.

Sobre estas buenas prácticas seleccionadas, se realizó a continuación un informe de investigación de las mismas, de acuerdo al siguiente esquema que vemos recogido en la tabla expuesta a continuación.

Tabla 1. Esquema de investigación en los estudios de caso.

Nombre del proyecto
Nombre de la organización responsable del proyecto
¿Cuáles son los principales objetivos del proyecto?
¿Cuál es la metodología del proyecto?
¿Qué actividades incluye el proyecto?
¿Cuáles fueron/son los resultados obtenidos?
¿Podría describir el contexto social del proyecto y los aspectos culturales en los que se centra?
Temporalización del proyecto
Agentes involucrados en el proyecto.
¿Qué entidades, instituciones, individuos colaboran en el proyecto?
¿Cómo diría que este proyecto contribuye al conocimiento intercultural y a la coexistencia social?
¿En qué sentido es innovador el proyecto (modalidad, objetivos, metodología...)?
¿Hasta qué punto están involucrados los inmigrantes en el proyecto?
¿Cuáles fueron los principales inconvenientes en la realización del proyecto (objetivos, contexto
social, subvención, participación...)?
¿En qué sentido opina que este proyecto podría ayudar a desarrollar otros proyectos en áreas
similares/el mismo área?
¿Podría proporcionar información de contacto con personas que de algún modo participasen en
el proyecto?
Por favor, incluya enlaces web relacionados con su proyecto

Los estudios de caso se han elaborado a partir de la información disponible en webs de los proyectos, revistas o documentos relacionados con la entidad y con el proyecto, así como a partir de la información obtenida de la realización de entrevistas a los responsables de los mismos. En cualquier caso, es necesario señalar que la selección final de los casos de estudios realizada dependió también del criterio de accesibilidad a la información requerida para la investigación. Este criterio determinó que el conjunto de final de casos seleccionados ${ }^{1}$ fuera el siguiente:

1 Toda la información relativa a estos casos de estudio está disponible en la página web del proyecto www. interculturemap.org. Asimismo, hay que tener en cuenta que un proyecto puede estar ubicado en más de un ámbito de conocimiento. 
TABLA 2: Proyectos seleccionados

\begin{tabular}{|c|c|}
\hline \multicolumn{2}{|r|}{ PROYECTOS ENMARCADOS EN EL ÁMBITO DEL EMPLEO } \\
\hline TEP & $\begin{array}{l}\text { Pacto territorial de empleo con objetivos específicamente dirigidos a la } \\
\text { inserción laboral de las personas inmigrantes. }\end{array}$ \\
\hline INTEG.R.A & $\begin{array}{l}\text { Proyecto dirigido a los refugiados, solicitantes de asilo y titulares del permiso } \\
\text { de residencia por motivos humanitarios. Aborda el aspecto laboral y el } \\
\text { alojamiento. www.integrarsi.anci.it/integra/ita/index.html }\end{array}$ \\
\hline $\begin{array}{l}\text { CENTROS } \\
\text { ARIWIT }\end{array}$ & $\begin{array}{l}\text { Proyecto dedicado a la formación profesional y la orientación laboral, } \\
\text { así como la inserción en el mercado de trabajo, dirigido a inmigrantes. } \\
\text { www.ariasociacion.org/ariwit1.html }\end{array}$ \\
\hline SOZE & $\begin{array}{l}\text { Proyecto desarrollado en la República Checa dirigidos principalmente a } \\
\text { refugiados y extranjeros. Ofrece asistencia legal, social y psicológica, así } \\
\text { como la puesta en marcha de actividades formativas, un centro multicultural } \\
\text { y una casa de acogida. http://soze.hyperlink.cz }\end{array}$ \\
\hline ECRE & $\begin{array}{l}\text { Desarrolla un pacto territorial de empleo enfocado exclusivamente a } \\
\text { refugiados y solicitantes de asilo. Pretenden lograr la protección e integración } \\
\text { de estos colectivos y la promoción de iniciativas coordinadas desde ONGs y } \\
\text { entidades e instituciones europeas. www.ecre.org/ }\end{array}$ \\
\hline $\begin{array}{l}\text { CANAL } \\
\text { SOLIDARIO }\end{array}$ & $\begin{array}{l}\text { Proyecto virtual que ofrece a los usuarios información y formación en } \\
\text { diferentes aspectos, resaltando aquellas temáticas relacionadas con el mercado } \\
\text { laboral. www.canalsolidario.org }\end{array}$ \\
\hline $\begin{array}{l}\text { ENTRA EN } \\
\text { XARXA }\end{array}$ & $\begin{array}{l}\text { Proyecto dirigido a la inserción sociolaboral de mujeres jóvenes inmigrantes } \\
\text { a través de cursos, orientación y seguimiento del itinerario personal de } \\
\text { inserción. Las participantes han elaborado una web del proyecto. } \\
\text { www.entraenxarxa.org }\end{array}$ \\
\hline CROCEVIA & $\begin{array}{l}\text { Ciclo de seminarios dirigido a profesionales que deben afrontar las situaciones } \\
\text { derivadas del fenómeno migratorio. www.fieri.it }\end{array}$ \\
\hline IASUK & $\begin{array}{l}\text { Proyecto preferentemente para la asesoría y representación legal de la } \\
\text { población inmigrante y solicitantes de asilo en cualquier materia. } \\
\text { Www.iasuk.org }\end{array}$ \\
\hline $\begin{array}{l}\text { PARTICIPACIÓN } \\
\text { SINDICAL EN } \\
\text { LOS PROCESOS } \\
\text { MIGRATORIOS }\end{array}$ & $\begin{array}{l}\text { Proyecto que pretende la participación de las organizaciones sindicales en los } \\
\text { procesos migratorios laborales y gestión de los flujos migratorios. } \\
\text { www.sindicatoseinmigracion.org }\end{array}$ \\
\hline $\begin{array}{l}\text { RED- } \\
\text { INTERLABORA } \\
\text { /ITINERARIOS } \\
\text { DE INSERCIÓN }\end{array}$ & $\begin{array}{l}\text { Acciones encaminadas a la inserción sociolaboral de los inmigrantes que } \\
\text { llegan a España como el aprendizaje del idioma, conocimiento del sistema } \\
\text { legal, acciones formativas, intermediación laboral, etc. www.cruzroja.es }\end{array}$ \\
\hline $\begin{array}{l}\text { CENTRO DE } \\
\text { ACOGIDA DE } \\
\text { MUJERES } \\
\text { INMIGRANTES }\end{array}$ & $\begin{array}{l}\text { Trabaja diversos campos de la calidad de vida. En el campo del empleo, desde } \\
\text { el Centro se llevan a cabo acciones de orientación laboral y formación en } \\
\text { aspectos concretos que atañen al empleo de las usuarias. }\end{array}$ \\
\hline CASA INSIEME & $\begin{array}{l}\text { Este proyecto trabaja de modo general los tres ámbitos de la Calidad de vida, } \\
\text { en cuanto al empleo facilita la creación de redes sociales para encontrar un } \\
\text { puesto de trabajo así como actividades formativas. } \\
\text { www.mappamondo.ra.it/progetto_casa_insieme.htm }\end{array}$ \\
\hline
\end{tabular}

\section{PROYECTOS ENMARCADOS DENTRO DEL ÁREA DE SALUD}

\begin{tabular}{|l|l|}
\hline \multicolumn{2}{|c|}{ PROYECTOS ENMARCADOS DENTRO DEL ÁREA DE SALUD } \\
\hline BAOBAB & $\begin{array}{l}\text { Este proyecto desarrolla su actividad principalmente en el área de alojamiento, } \\
\text { sin embargo también lleva a cabo acciones de carácter sanitario y relacionadas } \\
\text { con el empleo. www.erythros.org/progBaobab.asp }\end{array}$ \\
\hline NAFSIYAT & $\begin{array}{l}\text { El proyecto es un Centro de terapia intercultural, sus actividades sse dirigen } \\
\text { de forma específica a poblaciones minoritarias, trabajan ttemas de formación } \\
\text { relacionados con la terapia y la iinterculturalidad. www.nafsiyat.org.uk }\end{array}$ \\
\hline TAMPEP & $\begin{array}{l}\text { Proyecto que trabaja la prevención de enfermedades de transmisión sexual en } \\
\text { trabajadoras del sexo de población inmigrante. www.tampep.com }\end{array}$ \\
\hline
\end{tabular}




\begin{tabular}{|l|l|}
\hline CASA INSIEME & $\begin{array}{l}\text { Dentro de este proyecto se abordan aspectos relacionados con la salud y el } \\
\text { bienestar como cursos de relajación, charlas informativas sobre los recursos } \\
\text { existente en el territorio en el ámbito de la salud pública... } \\
\text { Www.mappamondo.ra.it/progetto_casa_insieme.htm }\end{array}$ \\
\hline $\begin{array}{l}\text { CENTRO DE } \\
\text { ACOGIDA PARA } \\
\text { MUJERES } \\
\text { INMIGRANTES }\end{array}$ & $\begin{array}{l}\text { Este proyecto además del campo laboral, desarrolla acciones relacionadas con } \\
\text { la Salud tanto de prevención como de promoción. }\end{array}$ \\
\hline $\begin{array}{l}\text { CONOCIENDO } \\
\text { NUESTRO }\end{array}$ & $\begin{array}{l}\text { En el campo de la salud, el acceso a recursos de salud a través de un } \\
\text { conocimiento mayor de los mismos es importante así como la influencia que } \\
\text { tiene en la salud mental y psicológica el sentirse integrado en la zona donde } \\
\text { se reside y contar con redes de apoyo. www.huelvaacoge.org }\end{array}$ \\
\hline IMISCOE & $\begin{array}{l}\text { Se centra en aspectos de investigación en el campo de la salud así como } \\
\text { en cuidados en este sentido. También elaboran marcos teóricos y posibles } \\
\text { aspectos de investigación en esta temática. www.imiscoe.org }\end{array}$ \\
\hline
\end{tabular}

\begin{tabular}{|l|l|}
\hline \multicolumn{2}{|c|}{ PROYECTOS ENMARCADOS DENTRO DEL ÁREA DE ALOJAMIENTO } \\
\hline INTEG.R.A & $\begin{array}{l}\text { Se aborda al área del alojamiento a través de iniciativas de intermediación } \\
\text { entre el mercado inmobiliario y los inmigrantes, creación de pisos tutelados, } \\
\text { asesoramiento sobre el acceso a viviendas de protección oficial, etc. } \\
\text { www.integrarsi.anci.it/integra/ita/index.html }\end{array}$ \\
\hline BAOBAB & $\begin{array}{l}\text { Puesta en marcha de un centro de acogida para refugiados, solicitantes de } \\
\text { asilo y titulares del permiso de residencia por motivos humanitarios. Son los } \\
\text { propios usuarios quienes gestionan el centro. } \\
\text { www.erythros.org/progBaobab.asp }\end{array}$ \\
\hline IASUK & $\begin{array}{l}\text { El proyecto Immigration Advisory Service dirigido a inmigrantes y solicitantes } \\
\text { de asilo, su actividad principal es el asesoramiento y la representación legal } \\
\text { de carácter gratuito. www.iasuk.org }\end{array}$ \\
\hline SOZE & $\begin{array}{l}\text { Es un proyecto que cuenta con una casa de acogida y un centro multicultural. } \\
\text { http://soze.hyperlink.cz }\end{array}$ \\
\hline $\begin{array}{l}\text { CONOCIENDO } \\
\text { NUESTRO } \\
\text { BARRIO }\end{array}$ & $\begin{array}{l}\text { En cuanto al alojamiento, no se encamina directamente a este ámbito pero sí } \\
\text { trabaja por una convivencia pacífica, tolerante. www.huelvaacoge.org }\end{array}$ \\
\hline
\end{tabular}

La realización de este proyecto no ha estado exento de dificultades, entre las que podemos señalar las siguientes. El no manejar algunos idiomas ha impedido poder llegar a cierta información. Esto también se ha visto impedido por falta de colaboración, bien por negativa o bien por omisión de respuesta a colaborar tanto en la elaboración de estudios de caso como en la cumplimentación del cuestionario. Resultó a veces complicado clasificar a proyectos y actores, así como distinguirlos, puesto que es común que un mismo proyecto cubra varias áreas de actuación y, en otros casos, actor y proyecto se confunden, ya que existen asociaciones que se dedican en exclusiva a un solo proyecto.

A raíz de este proyecto no sólo nos hemos acercado teóricamente a conceptos tan vigentes hoy en día y de tanta importancia como es la gestión, desde la interculturalidad, de la diversidad presente en nuestras sociedades, sino que hemos podido realizar estudios de caso concretos de experiencias específicas que pueden considerarse buenas prácticas en este sentido, ayudándonos a conceptualizar lo que podemos entender por Buena Práctica e Interculturalidad desde una base real y cercana a lo que realmente se está dando y produciendo, en este sentido, en las comunidades y sociedades hoy en día. 


\section{RESULTADOS: BUENAS PRÁCTICAS INTERCULTURALES EN EL ÁMBITO DE CALIDAD DE VIDA}

Nos resta aproximarnos al concepto de buenas prácticas. Para ello partimos de las conclusiones en torno a este concepto a la que los socios de este proyecto llegaron tras un encuentro preliminar, tomando en consideración además las definiciones de otros expertos (FEAPS, 2003). Una buena práctica vendría definida por las siguientes características. Ser innovadora, desarrollando soluciones nuevas y creativas para problemas comunes. Tener un impacto tangible y positivo sobre las condiciones de vida de aquellos a los que se dirige tanto como en las políticas sociales. Que pueda ser exportable y/o repetible: debe servir como marco de referencia a políticas o iniciativas nuevas o similares en contextos novedosos. Para ello debe contar con objetivos y planes de acción claros y bien definidos. Tener un efecto "sostenible" temporal y económicamente; una buena práctica debe contribuir a la erradicación estable de situaciones conflictivas, de necesidad o problemas concretos. Desarrollar la acción desde la profesionalidad y la interdisciplinariedad, contemplando la contribución de expertos y las necesidades formativas del propio personal. Permitir una participación activa de los usuarios en los procesos de actuación y cambio -en nuestro caso, esto se traduce en una implicación de la comunidad inmigrante-. Flexibles, toda buena práctica debe ser capaz de adaptarse a nuevas situaciones imprevistas. Evaluables, la evaluación debe diseñarse y planificarse desde que el proyecto o intervención comienza a gestarse, como parte inherente e indispensable del mismo.

Sin embargo, los proyectos seleccionados para nuestro estudio no sólo deberían ser buenas prácticas sino incorporar la dimensión intercultural en su desarrollo. Ello otorgaba a nuestro trabajo una dificultad mayor, ya que no todas las prácticas que se desarrollan de cara a la población de origen extranjero o con la diversidad son interculturales. De hecho, es relativamente reciente la aparición de tal término para definir intervenciones en contextos caracterizados por su diversidad.

Quizás, y a pesar de lo reciente del término, se ha abusado del mismo como adjetivo calificativo de proyectos e iniciativas que, al analizarl con mayor profundidad, se revelaban más propios de otros modelos de integración social. Esta proliferación de prácticas "interculturales" que carecen de una referencia teórica clara que defina lo intercultural han contribuido a favorecer una serie de ambigüedades en torno a este término que no han favorecido la difusión de este nuevo paradigma y modelo de integración social (Abdallach-Pretceille, 2001:34-38). Una de estas ambigüedades es la indisoluble relación que para muchos existe entre interculturalidad e inmigración, olvidando otras fuentes de diversidad que nos rodean y que también necesitan abordarse. Bien es cierto, como apuntan Vázquez (2005: 107) o García Castaño (2000: 211) que, con la inmigración, la diversidad se ha hecho más patente que nunca y su gestión ha comenzado a copar en mayor medida el discurso en las diferentes áreas de la intervención social y a diferentes niveles, pero eso no implica que la diversidad se agote en la misma. 
Desde nuestra óptica, cada uno de los proyectos elegidos para ser analizados debía cumplir no solo los requisitos de una buena práctica enumerados hasta ahora sino que, para ser calificados de interculturales, debían cumplir a su vez todo aquello que define una acción intercultural, constituyendo verdaderos ejemplos de buenas prácticas interculturales. A partir de la definición de las mismas, exponemos a continuación una aplicación de estos conceptos en las áreas que conforman la calidad de vida.

En relación al empleo, algunas de las características que nos permitirán catalogar una iniciativa o proyecto como buena práctica intercultural y que se recogen en los diferentes estudios de caso elaborados pueden ser la orientación y búsqueda directa de empleo, el acompañamiento en todo este proceso al colectivo inmigrante en la sociedad de acogida, como el caso del Centro de acogida para mujeres inmigrantes de ACCEM que, a través de la orientación, permite que las usuarias se hagan conscientes de las acciones que deben desarrollar para lograr la integración en el mercado laboral. De igual modo, el proyecto "Entra en Xarxa" presta también el servicio de orientación laboral, pero de un modo particular, ya que, aunque esta orientación se realiza en el propio centro de la entidad responsable, también se lleva a cabo mediante una web creada por las propias usuarias desde donde intercambian experiencias y se orientan entre ellas. La formación en aspectos profesionales, habilidades sociales, idioma y conocimiento legal del mercado de trabajo de la sociedad de acogida o la ayuda legal para la regularización de la situación administrativa son acciones que se realizan en la gran mayoría de los proyectos analizados en este campo.

La formación, una de las herramientas más empleadas desde la inserción laboral, abarca desde la enseñanza y alfabetización en el idioma de la sociedad de acogida -los proyectos SOZE en la República Checa y ECRE e INTEG. R.A en Italia, pueden servirnos de ejemplo- hasta la formación en habilidades sociales, conocimiento del funcionamiento legal del mercado laboral, cursos técnico-profesionales específicos, como los que desarrollan los proyectos Centros Integrales ARI-WIT en España o "Casa Insieme", en la ciudad italiana de Ravenna. La formación se convierte también en el instrumento estrella cuando se trabaja con la población autóctona. Así, por ejemplo, los seminarios CROCEVIA en Turín o los de la "Fundación Ismu" en Milán destinados a profesionales que trabajan en contextos de creciente multiculturalidad. En este sentido, proyectos como el INTEG.R.A tienen estructurado todo un plan de formación destinado a sus propios trabajadores. Entre las otras muchas acciones que llevan a cabo, cabe destacar la ayuda y asesoramiento legal prestado en este campo (empleo) por parte del proyecto IASUK en el Reino Unido, el objetivo de la mejora de las condiciones de empleo del colectivo inmigrante del proyecto "Participación Sindical" y la elaboración del proyecto e itinerario personal de inserción laboral, contemplando y fomentando el autoempleo y la iniciativa empresarial, como el que lleva a cabo la red "Interlabora" y "Entra en xarxa" en Barcelona.

Si bien es cierto que dentro de este área la gestión de la diversidad se ve reducida por el esfuerzo del inmigrante/extranjero a adaptarse a las condiciones de trabajo de un mercado laboral que presenta unas características legales y culturales claramente definidas, también lo es que pueden tomarse en con- 
sideración por parte de los agentes empleadores algunos aspectos que harían posible una gestión de la diversidad beneficiosa para todos. Algunos de estos aspectos pueden ser la negociación del calendario festivo atendiendo a las diferencias culturales de los trabajadores, la conciliación de tiempos de trabajo y formas de organización del mismo propias de la cultura e identidad organizacional con la propia de los trabajadores... Nadie dice que sea fácil hacerlo, lo que sí debe quedar bien asentado es la idea de que estos esfuerzos no redundan en un peor rendimiento o una baja productividad por parte de los trabajadores y por ende de las empresas.

En el ámbito de la salud, hablar de interculturalidad supone un extenso debate dada la milenaria relación entre cultura y salud, mucho más controvertida cuando la relación se convierte en tríada y aparece en el campo de juego la ciencia. Los conceptos, creencias y nociones en torno a la salud, la enfermedad y los cuidados paliativos están tremendamente influenciados por aspectos culturales. Por ello, es necesario el abordaje intercultural que garantice una atención sanitaria de calidad por parte de los profesionales de la salud, especialmente de aquellos que desarrollen su profesión en contextos plurales y diversos (Lerín, 2004: 113-114). El proyecto NAFSIYAT en Londres, por ejemplo, trabaja para que los terapeutas desarrollen su profesión teniendo presente la realidad cultural del paciente, ejecutan acciones de sensibilización y formación en este campo, centrándose principalmente en la terapia intercultural.

Aún así, algunas pautas que pueden conducirnos a considerar en este ámbito una acción como buena práctica intercultural son aquellas que siguen el camino de la consideración de los aspectos culturales y la diversidad en esta área desde el momento en el que comienzan a planificarse las intervenciones. Desde esta óptica, pueden catalogarse como interculturales aquellos proyectos en los que se lleven a cabo las siguientes acciones: adecuar la atención sociosanitaria a la realidad de la inmigración - una realidad que es susceptible de ser experimentada como elemento desestabilizador de la salud (tanto física como psicológica) de los individuos-, adecuar igualmente el tratamiento teniendo presente el bagaje cultural y el origen de los individuos (factores de riesgo, etc.) y elaborar programas de prevención adecuados a las necesidades reales y que faciliten la adaptación al nuevo entorno.

Desde el proyecto TAMPEP, se desarrollan acciones en varios países europeos de prevención del contagio de enfermedades de transmisión sexual en trabajadoras del sexo inmigrantes a través del conocimiento y el trabajo directo con ellas, acciones de educación en salud, etc., todo ello teniendo presentes las condiciones socio-culturales de las mismas. A su vez, el abordaje se realiza teniendo en cuenta los aspectos sociales, psicológicos y legales de cada situación. La prevención se desarrolla junto con acciones de promoción de la salud en el Centro de acogida para mujeres inmigrantes de ACCEM, dirigiendo ambas acciones no sólo hacia las propias usuarias del centro sino que también reciben formación que les facilita desarrollar acciones saludables con sus hijos en el nuevo medio en el que se encuentran, respetando siempre las particularidades culturales y personales de cada una de ellas. Otras acciones desarrolladas en el campo de la salud desde diversos proyectos, pueden ser la realización de talleres y cursos de técnicas relacionadas con el bienestar y la relajación, formación relacionada con la articulación del sistema sanitario de la sociedad 
de acogida -como en el caso de "Casa Insieme"- y aspectos relacionados con la investigación en migración-salud, los factores que influyen en esta interacción y el modo de abordar desde la salud la diversidad cultural existente en el proyecto IMISCOE.

Otros aspectos que podrían llevarse a la práctica y que se encontrarían en la línea de conseguir una buena práctica intercultural en materia de salud serían el contar con indicaciones en varios idiomas en todos los centros sanitarios, así como con mediadores y traductores que permitan un trato óptimo del personal sanitario con el paciente salvando barreras lingüísticas y culturales que garanticen el entendimiento y comprensión o, como propone Alarcón (2003:1064), negociar y validar interculturalmente un modelo de salud-enfermedad para un contexto plural y diverso determinado que considere aspectos de las diferentes visiones que se manejan por profesionales y usuarios.

Definir y clarificar la relación entre interculturalidad y alojamiento en la realidad cotidiana o en los contextos actuales y poder sugerir indicadores de buenas prácticas interculturales resulta complicado. Esta relación lleva inherente la particularidad de que el alojamiento es una de las necesidades básicas que debe ser cubierta para facilitar, con posterioridad, una integración plena en la sociedad de acogida. Esto nos lleva al terreno de definir esta relación en términos de practicidad (de lo que cotidianamente se vive en comunidades de vecinos, en barrios...) y entender que, aunque la adaptación debe ser mutua, no cabe duda de que un mayor esfuerzo de adaptación corresponde a la persona que se incorpora a otro territorio u espacio.

La gran disyuntiva en este área es dilucidar dónde comienza aquí la acción que pueda llamarse "intercultural", cuál debe ser su objeto y cuáles sus destinatarios. Cabe preguntarnos qué acción es más intercultural: aquélla que se limita a buscar o facilitar el alojamiento -entendido como el espacio físico que habitar- o la que trabaja en una comunidad para enseñarle a vivir la diversidad que debe afrontar dada su reciente naturaleza multicultural, como en el caso del proyecto "Conociendo nuestro barrio", que trabaja por la integración y convivencia, en todos los ámbitos, de la población inmigrante y la autóctona en un barrio concreto de la ciudad de Huelva, en el que la inmigración ha tenido gran incidencia (con el aumento de las relaciones interculturales). Desde nuestro punto de vista, ambas intervenciones pueden llevarse a la práctica en clave intercultural si están gestados desde la constatación de la diversidad, la consideración del lugar que los actores y beneficiarios ocupan en la estructura social y las condiciones y relaciones de desigualdad que se generan a partir de lo anterior.

De ahí que un programa de intervención cuyo objetivo sea la creación de un centro de acogida temporal de inmigrantes, como ocurre en el Centro BAOBAB, que gestiona la asociación "Erythros" en Roma, con la particularidad de que trabaja con el colectivo de refugiados y solicitantes de asilo, o el Centro de acogida para mujeres inmigrantes de ACCEM, pueda considerarse como ejemplo de buena práctica intercultural si más allá de proporcionar alojamiento, tiene entre sus objetivos el facilitar la integración de sus usuarios en la comunidad en la que se inserta, con acciones que en estos y otros proyectos se plasman en servicios como el asesoramiento social y legal (IASUK, SOZE, 
BAOBAB. . .), atención médica, tratamiento del tiempo de ocio de los individuos así como el apoyo y orientación de escolarización de los menores desde el Centro de ACCEM y la autogestión del centro por los propios usuarios en el caso del Centro BAOBAB. Estas acciones van encaminadas a dotar de autonomía y facilitar la integración social de los individuos. Y trabaja, además, desde un doble frente: los usuarios del centro y el resto de la comunidad. Así, por ejemplo, el Centro BAOBAB, que cuenta con espacios de participación abiertos al resto de la comunidad, como un restaurante o un punto de encuentro intercultural con sala de reuniones, radio y exposiciones, o la implicación de los inmigrantes en la conservación de los espacios comunes de la comunidad.

Una agencia de intermediación inmobiliaria puede parecer a simple vista que no aporte nada a la difusión de la interculturalidad, si se limita a lo meramente administrativo y burocrático que tiene dicha actividad. Sin embargo, también creemos que es posible trabajar desde aquí en clave intercultural si se establece, por ejemplo, un estándar mínimo de calidad que debe cumplir la vivienda, si se tiene en cuenta una distribución geográfica en los patrones de asentamiento que no favorezca la "ghetización" de los inmigrantes y extranjeros, o si facilita y acerca el uso y disfrute de los recursos en materia de vivienda pública a estos colectivos en condiciones de igualdad con el resto de la sociedad de acogida. Una agencia para la vivienda, contemplada por ejemplo en el proyecto INTEG.R.A, trabaja por la consecución de todos los objetivos que se han expuesto anteriormente e intenta conseguir con ello el cambio de políticas sociales que favorezca un mejor acceso, y de calidad, a la vivienda por parte de la población inmigrante.

\section{CONCLUSIÓN}

Todos estos principios esbozados en el concepto de una buena práctica tienen su aplicación en la perspectiva intercultural, teniendo presentes los principios básicos de una buena Práctica y aquellos propios de la noción de interculturalidad y acción intercultural. Se trataría de una práctica que siendo flexible, sostenible, exportable, evaluable..., fomentara el diálogo cultural, el intercambio y la convivencia dinámica de individuos de diversas culturas, entendiendo la diferencia como aspecto enriquecedor, y fomentando la celebración de puntos comunes; favoreciendo con ello la inclusión en las instituciones nucleares de la sociedad de acogida (como pueden ser la educación, sanidad, empleo, vivienda, etc.) y respetando en el proceso de adaptación tanto la libertad y la identidad cultural propia del individuo como las normas culturales, legales, civiles y éticas básicas de la sociedad de acogida.

\section{REFERENCIAS BIBLIOGRÁFICAS}

ABDALLAH-PRETCEILLE, M. (2001). "El Interculturalismo en perspectiva” en La educación intercultural. Icaria: Barcelona, pp 95-114.

ALARCÓN, A.M; ALDO, H; NEIRA, J (2003). "Salud Intercultural: Elementos para la construcción de sus bases conceptuales”. En Revista Médica de Chile v. 131 n. 9. 
ARPINI, A. (2005) "Ética Intercultural” en Ciencias Sociales On line. Chile: Universidad Viña del Mar. vol. 2, n. 1, pp 67-74.

BLANCO, A. (1988) "Calidad de Vida" en Reyes, R.: Terminología Cientifico Social: Aproximación Crítica. Anthropos: Barcelona, pp 63-66

BÚRDALO, H. (2006) "La inmigración como riqueza" en Educación y Futuro Digital, en http:// www2.cesdonbosco.com/revista/articulos2006/junio06/helenaburdalo.p df. Consulta 12/I/07.

JORDÁN SIERRA, J.A. (2004) "Educar para la convivencia intercultural” en Educación y desarrollo. En http://www.infosolidaria.org/verarticulo.php?idarticulo=779\&idautor=94\&id version $=0$ \&ididioma $=1$. Consulta 26/X/06.

SHALOCK, R.; BROWN, I. y cols. (2005) “La conceptualización, medida y aplicación de calidad de vida en personas con discapacidades intelectuales: Informe de un panel internacional de expertos" en Siglo Cero. vol. 33 n ${ }^{\circ}$, pp 5-14.

TRIANDAFYLLIDOU, A (2005) "Nuevos retos para Europa, migración, seguridad y derechos de ciudadanía" en Revista CIDOB d'Afers Internacional, $\mathrm{n}^{\circ}$ 69, pp 39-59.

VÁZQUEZ AGUADO, O. (2005) "La competencia intercultural y las habilidades de intervención en mediación social intercultural" en Manual de Atención social al inmigrante. Córdoba: Almuzara, pp 107-125. 